\title{
A PROOF OF THE INVARIANT MEAN-VALUE THEOREM ON ALMOST PERIODIC FUNCTIONS
}

\author{
HUKUKANE NIKAIDÔ
}

1. Several procedures have been devised to establish the existence of an invariant mean for almost periodic functions on a group (see $[1 ; 3 ; 4 ; 5]) .^{1}$ The object of the present short note is to prove this basic result concerning such functions in another way. Throughout what follows the formula $\phi(x): A \rightarrow B$ regarding a function $\phi(x)$ will be used to imply that $\phi(x)$ is defined on a set $A$ and has a subset of $B$ as its range.

2. Let $G$ be a group and $E$ be a complete, locally convex, topological linear space. In accordance with A. Weil's method (see [6]) we can associate a compact topological group $\bar{G}$ with $G$ so that every almost periodic function $\phi(x): G \rightarrow E$ in the sense of Bochner-von Neumann (see [1]) can be written in the following fashion: $\phi(x)$ $=\phi(\theta(x))$, where $\theta(x): G \rightarrow \bar{G}$ is a fixed homomorphism, which is independent of $\phi$, and $\bar{\phi}(\bar{x}): \bar{G} \rightarrow E$ is a continuous function corresponding to $\phi$. Therefore, without loss of generality, we may regard $G$ itself as a compact topological group and $\phi(x)$ as a continuous function on it. Denote by $E^{G}$ the linear space consisting of all continuous functions $\phi(x): G \rightarrow E$, and by $\left\{U_{\lambda} \mid \lambda \in \Lambda\right\}$ a fundamental system of neighborhoods around the zero of $E$. It is known that a complete, locally convex, linear topology is introduced into $E^{G}$ by adopting $\left\{V_{\lambda} \mid \lambda \in \Lambda\right\}$ as a fundamental system of neighborhoods around the zero of $E^{G}$, where $V_{\lambda}=\left\{\phi \mid \phi(G) \subset U_{\lambda}\right\}$. Set $L_{\phi}=\{\phi(a x) \mid a \in G\}$ for a $\phi \in E^{G}$, and denote by $K_{\phi}$ the closure of the convex hull of $L_{\phi}$; then we obtain a compact convex subset $K_{\phi}$ of $E^{G}$. The result to be proved is

Invariant Mean-Value Theorem. For every $\phi \in E^{\sigma}, K_{\phi}$ contains a constant-valued function.

To this end we shall first establish

Fixed-Point Theorem. Let $K$ be a compact convex subset lying in a locally convex, topological linear space $E$. Given a continuous mapping $\Phi(x): K \rightarrow K$, which is convex linear, i.e. $\Phi\left(\alpha_{1} x_{1}+\alpha_{2} x_{2}\right)=\alpha_{1} \Phi\left(x_{1}\right)$ $+\alpha_{2} \Phi\left(x_{2}\right)$ for $x_{1}, x_{2} \in K, \alpha_{1}, \alpha_{2} \geqq 0, \alpha_{1}+\alpha_{2}=1$, so $\Phi$ admits fixed-points.

Received by the editors July 8, 1954 .

${ }^{1}$ Besides the articles referred to herewith a useful tool for this purpose is the mean ergodic theorem achieved by F. Riesz, K. Yoshida, S. Kakutani and other authors. 
Proof. The result could be obviously obtained by applying Tychonoff's fixed-point theorem without referring to convex-linearity. We shall give, however, a much simpler proof corresponding to this additional assumption. Set indeed $\Psi(x)=x-\Phi(x)$. The mapping $\Psi(x): K \rightarrow E$ is also continuous and convex linear so that $\Psi(K)$ is a compact convex subset. If $0 \notin \Psi(K)$, there exists a continous linear functional $f$ such that $f(x)>0$ on $\Psi(K)$. Choose one point $\hat{x} \in K$ with $\min _{x \in K} f(x)=f(\hat{x})$, and set $\hat{y}=\hat{x}-\Phi(\hat{x})$. Hence $f(\hat{y})=f(\hat{x}-\Phi(\hat{x}))$ $=f(\hat{x})-f(\Phi(\hat{x})) \leqq 0$, leading to a contradiction. Thus $0 \in \Psi(K)$, which was to be shown.2

Proof of the invariant mean-Value theorem. Denote by (S) $=\left\{A_{\gamma} \mid \gamma \in \Gamma\right\}$ the family of all finite subsets $A_{\gamma}$ of $G$. Let $A_{\gamma}=\left\{a_{i} \mid i=1,2, \cdots, m\right\}$, and consider the mapping $\psi \rightarrow \Phi(\psi)$ $=(1 / m) \sum \psi_{a_{i}}: K_{\phi} \rightarrow K_{\phi}$, where $\psi_{a}(x)=\psi(a x)$. This mapping is obviously continuous and convex-linear so that there exists a point $\psi$ in $K_{\phi}$ with $\psi=\Phi(\psi)$. Denote further by $\mathfrak{B}=\left\{B_{\delta} \mid \delta \in \Delta\right\}$ the family of all finite subsets $B_{\delta}$ of the conjugate space $E^{*}$ of $E$. Setting $F(y)=\left(f_{1}(y)\right.$, $\left.f_{2}(y), \cdots, f_{n}(y)\right)$ for a $B_{\delta}=\left\{f_{j} \mid j=1,2, \cdots, n\right\}$, we obtain a continuous linear mapping $F(y): E \rightarrow E_{n}$, where $E_{n}$ is an $n$-dimensional Euclidean space. For a point $z \in E_{n}$ let $\|z\|$ denote the usual Euclidean norm. As $\psi(x)=(1 / m) \sum \psi_{a_{i}}(x)$ for all $x \in G$, we have also $F(\psi(x))$ $=(1 / m) \sum F\left(\psi_{a_{i}}(x)\right)$ for all $x \in G$.

Choose a point $\hat{x} \in G$ such that $\max _{x \in a}\|F(\psi(x))\|=\|F(\psi(\hat{x}))\|$.

Then $\|F(\psi(\hat{x}))\| \leqq(1 / m) \sum\left\|F\left(\psi_{a_{i}}(\hat{x})\right)\right\|=(1 / m) \sum\left\|F\left(\psi\left(a_{i} \hat{x}\right)\right)\right\|$ so that $\|F(\psi(\hat{x}))\|=\left\|F\left(\psi_{a_{i}}(\hat{x})\right)\right\|(i=1,2, \cdots, m)$, because $\|F(\psi(\hat{x}))\|$ $\geqq\left\|F\left(\psi\left(a_{i} \hat{x}\right)\right)\right\|(i=1,2, \cdots, m)$. Therefore the $m+1$ points $F(\psi(\hat{x}))$, $F\left(\psi_{a_{i}}(\hat{x})\right)(i=1,2, \cdots, m)$ together lie on a sphere (possibly of radius zero). The equation $F(\psi(\hat{x}))=(1 / m) \sum F\left(\psi_{a_{i}}(\hat{x})\right)$ implies then that $F(\psi(\hat{x}))=F\left(\psi_{a_{i}}(\hat{x})\right)(i=1,2, \cdots, m)$.

Set now $N_{\gamma, \delta}=\left\{(\psi, x) \mid f(\psi(x))=f\left(\psi_{a}(x)\right), a \in A_{\gamma}, f \in B_{\delta}\right\}$.

For every $(\gamma, \delta) \in \Gamma \times \Delta, N_{\gamma, \delta}$ is closed in the cartesian product $K_{\phi} \times G$, since the mapping $(\psi, x) \rightarrow \psi(x): K_{\phi} \times G \rightarrow E$ is continuous. Thus we obtain a family of closed subsets $\left\{N_{\gamma, \delta} \mid(\gamma, \delta) \in \Gamma \times \Delta\right\}$ in $K_{\phi} \times G$, which admits in addition the finite intersection property. In view of the compactness of $K_{\phi} \times G$ this implies that $\bigcap_{(\gamma, \delta) \in \Gamma \times \Delta} N_{\gamma, \delta} \neq \phi$. Choose a point $(\hat{\psi}, \hat{x})$ belonging to all $N_{\gamma, \delta}$ 's. We have thus $f(\hat{\psi}(\hat{x}))$ $=f\left(\widehat{\psi}_{a}(\hat{x})\right)$ for any $a \in G, f \in E^{*}$, so that $\widehat{\psi}(\hat{x})=\widehat{\psi}(a \hat{x})$ for any $a \in G$, yielding a constant-valued function $\widehat{\psi} \in K_{\phi}$, as was to be shown.

The uniqueness of mean-value is easily seen in the same fashion as usual.

2 Cf. Appendice to [2]. 
3. A direct application of the theorem will be described below. Let $G$ be a compact topological group and $E$ be the Banach space consisting of all continuous numerical functions $f$ on $G$ with

$$
\|f\|=\sup _{x \in G}|f(x)|
$$

as the norm. As is known, every regular Borel measure $\mu$ over $G$ can be regarded as a point in the conjugate space $E^{*}$ of $E$ and vice versa.

Choose an arbitrary $\mu \in E^{*}$. Defining $\mu_{a}(f)=\mu\left(f_{a}\right), \phi(x)=\mu_{x}$, we have a continuous function $\phi(x): G \rightarrow E^{*}$, the continuity being viewed from the standpoint of the weak topology of $E^{*}$ induced by $E$, so that the invariant mean-value theorem applies to it. Hence $K_{\phi}$ contains a constant-valued function $\widehat{\psi}(x) \equiv \widehat{\mu}$, which represents an invariant measure over $G$. If one starts from a positive, nontrivial $\mu$, the resulting measure is nothing but a Haar measure. Furthermore, if the starting measure is in particular such a measure as assigns to a point in $G$ the probability measure 1, one obtains the classical result: if a finite number of numerical almost periodic functions $f(x)$ on a group $G$ are given, their respective means can be uniformly and simultaneously approximated by functions of the form: $\sum \alpha_{i} f\left(a_{i} x\right)$, $\alpha_{i} \geqq 0, \sum \alpha_{i}=1$ with the same $a_{i}$ 's and $\alpha_{i}$ 's.

4. In contrast with the argument of the example in the appendix to [2], where the commutativity assumption is basic, intensive use was made of the compactness of $G$ in the fixed-point method described above to overcome the main difficulty due to the situation that $G$ is not necessarily abelian.

\section{REFERENCES}

1. S. Bochner and J. von Neumann, Almost periodic functions in groups. II, Trans' Amer. Math. Soc. vol. 37 (1935).

2. N. Bourbaki, Eléments de mathématiques. Book V. Espaces vectoriels topologiques, 1953, especially Appendice.

3. S. Iyanaga and K. Kodaira, On the theory of almost periodic functions in a group, Proc. Imp. Acad. Tokyo vol. 16 (1940).

4. W. Maak, Eine neue Definition der fastperiodischen Funktionen, Abh. Math. Sem. Hamburgischen Univ. vol. 11 (1935).

5. J. von Neumann, Almost periodic functions in a group. I, Trans. Amer. Math. Soc. vol. 36 (1934).

6. A. Weil, L'intégration dans les groupes topologiques et ses applications, 1940.

Tokyo College of Science 